\title{
Epitranscriptomic $\mathbf{N}^{6}$-methyladenosine modification is required for direct lineage reprogramming into neurons
}

\author{
Hwan Choi ${ }^{a}, 1$,Soonbong Baek ${ }^{b, 1}$, Byounggook Choa, Siyoung Kimª, Junyeop Kimª, \\ Yujung Chang ${ }^{\mathrm{a}}$, Jaein Shina, Jongpil Kim ${ }^{\mathrm{a}, \mathrm{c}^{*}}$
}

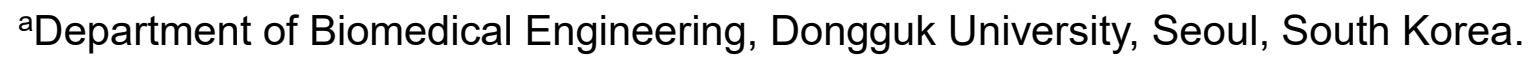

${ }^{\mathrm{b}}$ Developmental Disorders \& Rare Diseases Research Group, Korea Brain Research Institute, Daegu 40162, Korea, Republic.

'Department of Chemistry, Dongguk University, Seoul, South Korea.

${ }^{1}$ These authors contributed equally to this work.

\section{${ }^{*}$ Correspondence}

JONGPIL KIM, Ph.D.

Distinguished Professor

Dept of Chemistry

Dept of Biomedical Engineering

Director, Center for Regenerative Medicine

Director, BK21Plus team for Regenerative Medicine

Dongguk University,

Seoul, Korea

Tel: 82-02-2260-3213

Cell: 82-010-4013-3685

Email: 1k2316@gmail.com, jpkim153@dongguk.edu 
Supplementary figure 1

A
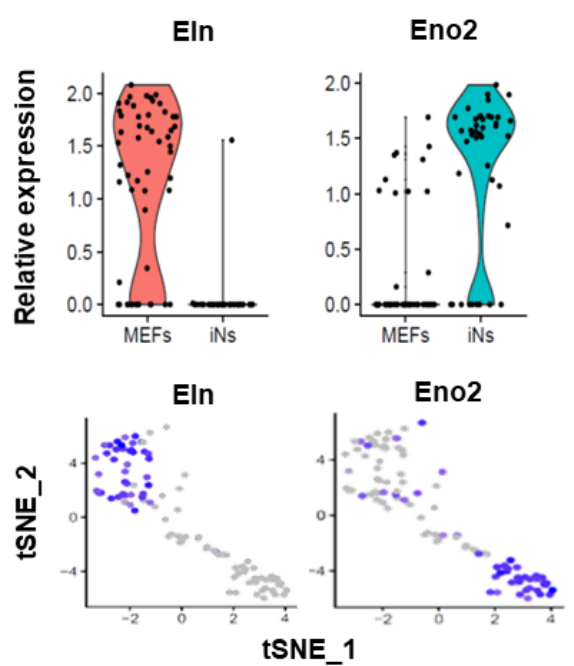

B

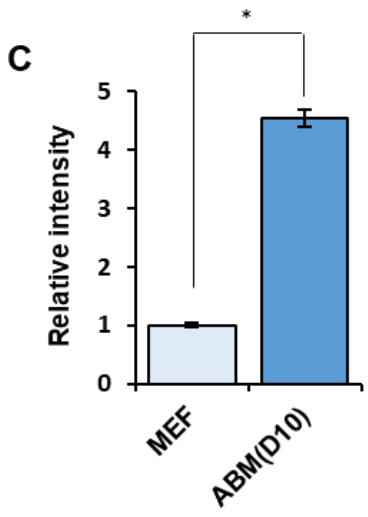

Supplementary figure 1. Expression of Mettl3 in direct neuronal reprogramming.

(A) Violin plots (Top) and t-distributed stochastic neighbor embedding (t-SNE) (Bottom) show the distribution of fibroblast gene elastin (Eln) and Mettl3 in MEFs and iNs. Three independent experiments were performed. Data represent the mean $\pm \mathrm{SEM},{ }^{*} P<0.05$ $(\mathrm{n}=3)$, Student's t-test. (b) Western blot analysis showing a change in the expression level of Mettl3 protein at day 5 after ABM transduction. (C) Densitometric analysis showing the fold increase of Mettl3 protein in ABM-treated MEFs. Three independent experiments were performed. Data represent the mean \pm SEM. ${ }^{*} P<0.05(\mathrm{n}=3)$, Student's t-test. 


\section{Supplementary figure 2}

A

$\square$ 5'UTR $\square$ TSS $\square$ CDS $\square$ TTS + 3'UTR

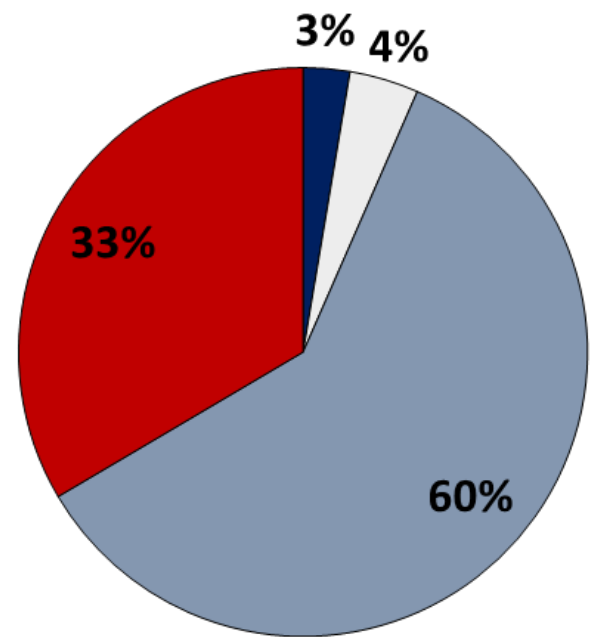

Supplementary figure 2. Distribution of $m^{6} A$ peaks in direct neuronal reprogramming. (A) Venn diagram of the frequency of $m^{6} \mathrm{~A}$ peak in genes, divided into five distinct regions (5'-UTR, transcription start site (TSS), coding sequence (CDS), transcription termination site (TTS), and 3'-UTR) 


\section{Supplementary figure 3}

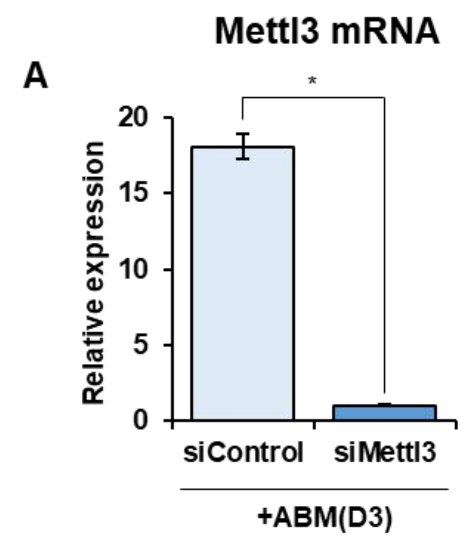

B

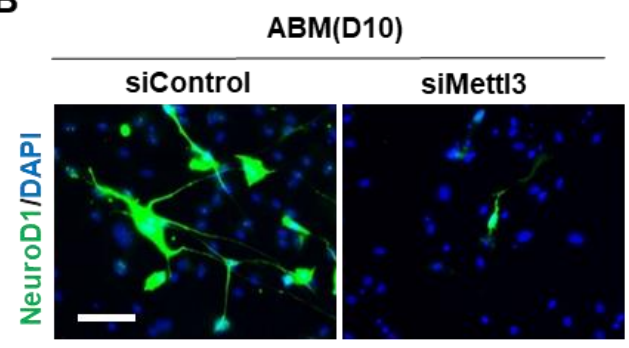

C

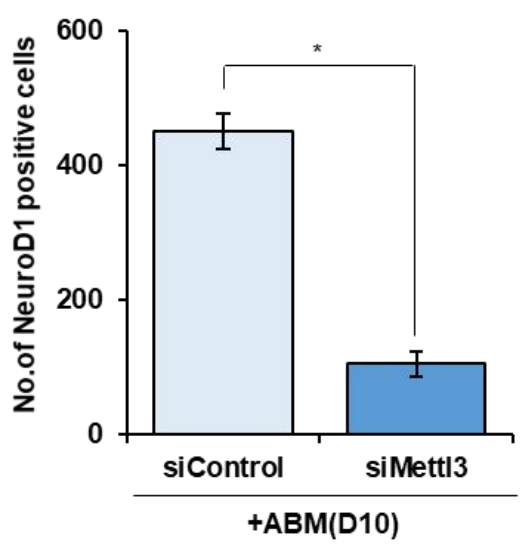

Supplementary figure 3. Mett|3 is required for direct neuronal reprogramming.

(A) Quantification of the relative levels of Mett|3 after siMett|3 treatment. Results are expressed as fold change relative to GAPDH. Three independent experiments were performed. Data represent the mean \pm SEM. ${ }^{\star} P<0.05(\mathrm{n}=3)$, Student's t-test. (b) Representative immunofluorescent images and (C) quantification of NeuroD1-positive cells in ABM-transduced MEFs with Mettl3 knockdown. Three independent experiments were performed. Data represent the mean \pm SEM. ${ }^{\star} P<0.05(\mathrm{n}=3)$, Student's t-test. Scale bar: $100 \mu \mathrm{m}$. 


\section{Supplementary figure 4}

A

\begin{tabular}{|cc|}
\hline Gene symbol & Peak score \\
\hline Btg2 & 65.92 \\
\hline Cdh11 & 40.91 \\
\hline Pten & 21.97 \\
\hline Foxo3 & 25.76 \\
\hline Lemd2 & 15.15 \\
\hline Taf1 & 14.02 \\
\hline Atoh8 & 13.26 \\
\hline Ep300 & 12.88 \\
\hline Neurog2 & 6.82 \\
\hline
\end{tabular}

B
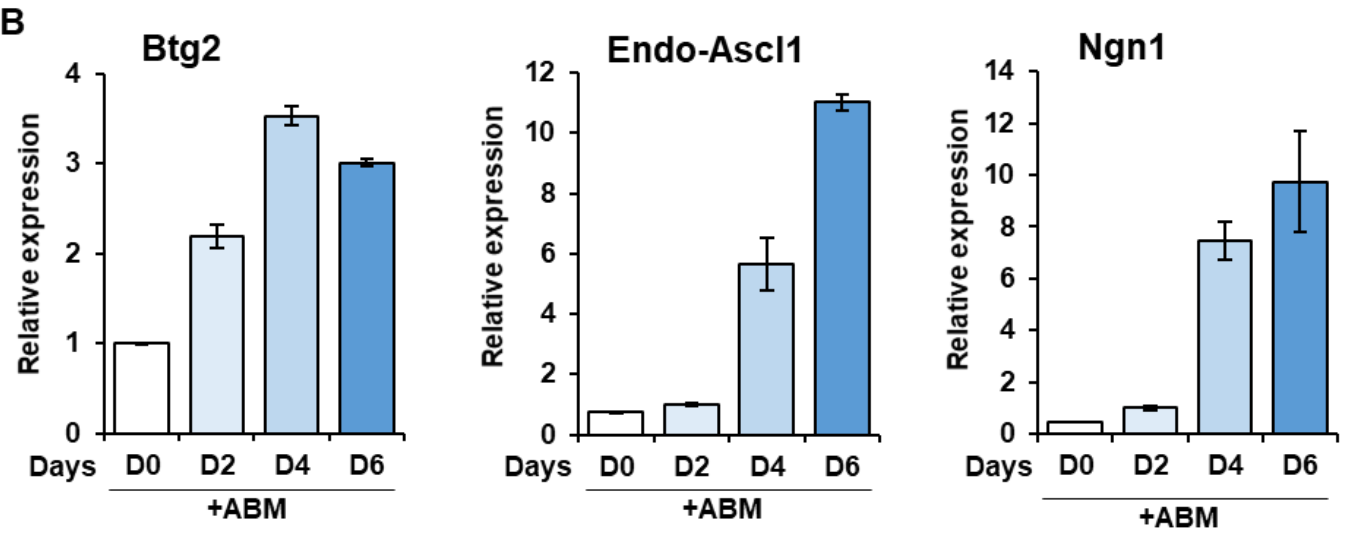

Supplementary figure 4. Expression of Btg2 in direct neuronal reprogramming.

(A) The top-9 most enriched $\mathrm{m}^{6} \mathrm{~A}$ RIP-seq-based target transcripts of neuronal genes.

(B) Time course of Btg2 and proneural genes (Ascl1 and Neurogenin1) during iNs generation by qRT-PCR analysis. Three independent experiments were performed.

Data represent the mean \pm SEM. 


\section{Supplementary figure 5}

A
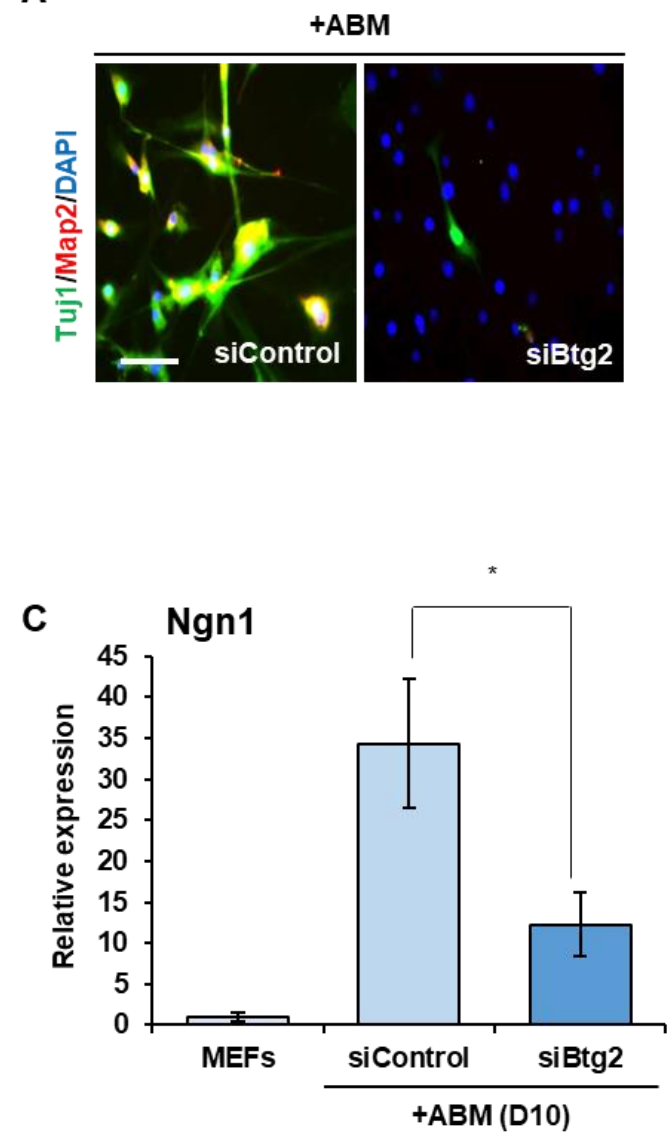

B
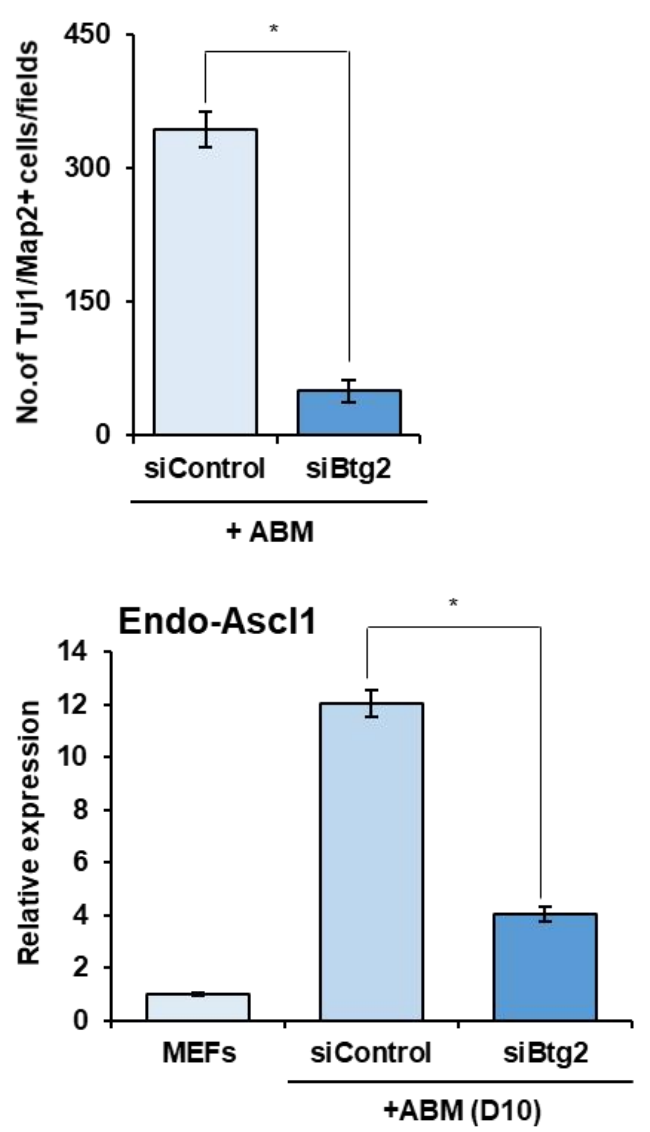

Supplementary figure $\mathbf{5}$. Btg2 is required for direct neuronal reprogramming. (A) Btg2 knockdown in ABM-tranduced cells was confirmed by Immunofluorescent images and (B) quantification of Tuj1- and Map2-positive cells in ABM with siControlor siBtg2-transduced MEFs. Data represent the mean \pm SEM. ${ }^{*} P<0.05(n=3)$, Student's t-test. Scale bar: $100 \mu \mathrm{m}$. (C) mRNA expression of proneural genes (Ascl1 and Neurog1) in ABM with siControl- or siBtg2-transduced MEFs. Three independent experiments were performed. Data represent the mean \pm SEM. ${ }^{*} P<0.05(n=3)$, Student's t-test. 
Supplementary figure 6

A

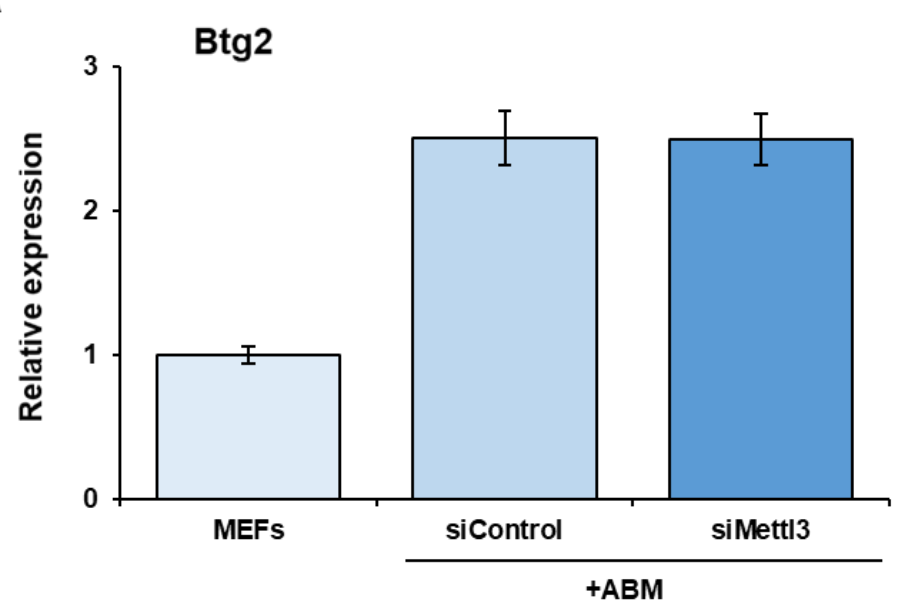

Supplementary figure 6. Quantification of Btg2 transcripts in direct neuronal reprogramming upon Mettl3 knockdown. (A) mRNA expression of Btg2 in ABM with siControl- or siMettl3-transduced MEFs. Three independent experiments were performed. Data represent the mean \pm SEM. 


\section{Supplementary figure 7}

A

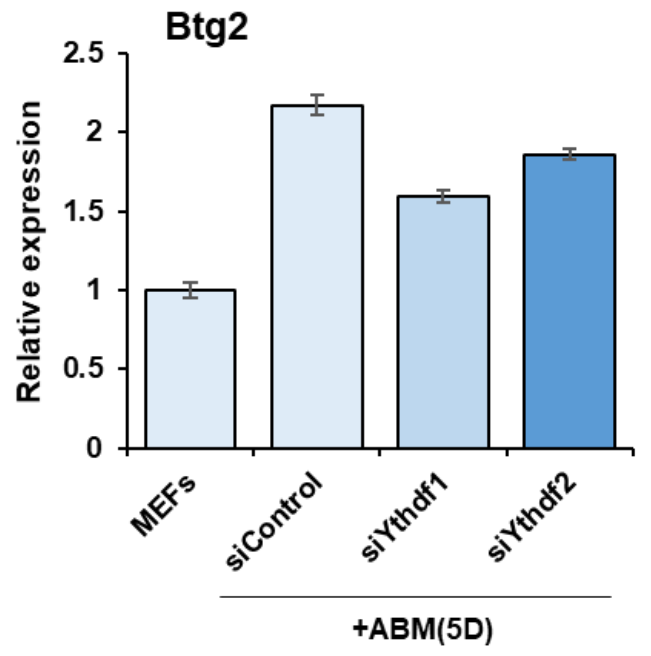

B
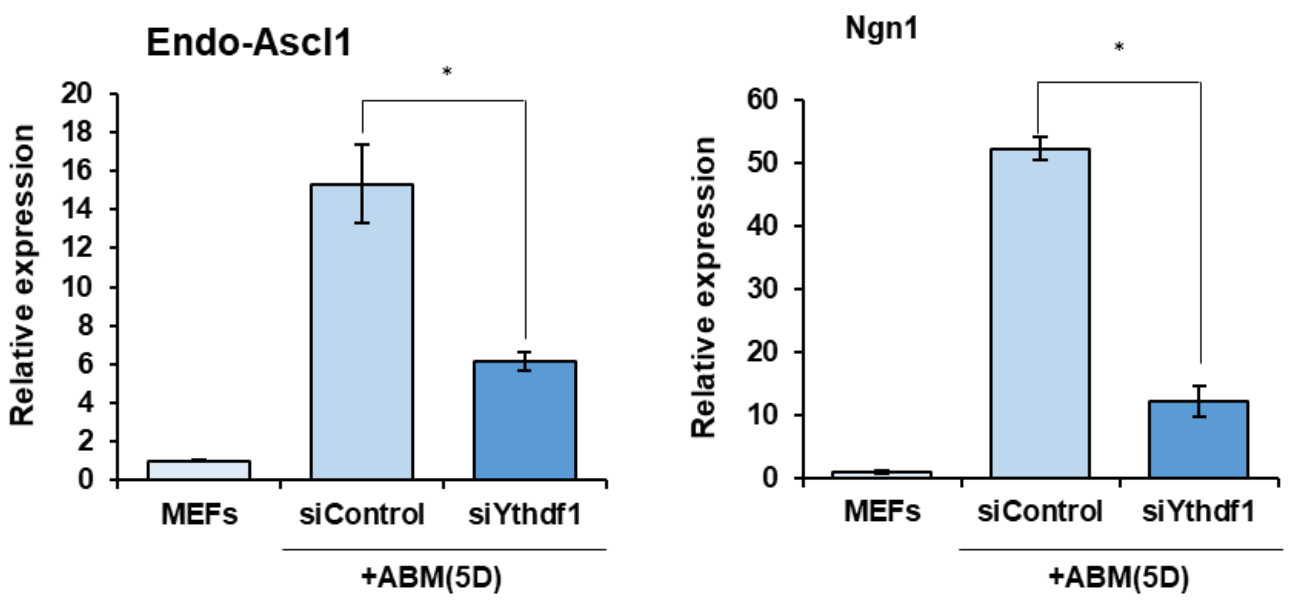

Supplementary figure 7. Ythdf1 is required for direct neuronal reprogramming.

(A) qRT-PCR analysis of Btg2 at 5 days after ABM with siControl-, siYthdf1-, or siYthdf2-transduced MEFs. Three independent experiments were performed. Data represent the mean \pm SEM. (B) mRNA expression of Endo-Ascl1 and Neurogenin1 5 days after ABM with siControl- and siYthdf1-transduced MEFs. Three independent experiments were performed. Data represent the mean \pm SEM. ${ }^{*} P<0.05(n=3)$, Student's t-test. 